\title{
Q Estimation of Seismic Data Using the Generalized S-transform
}

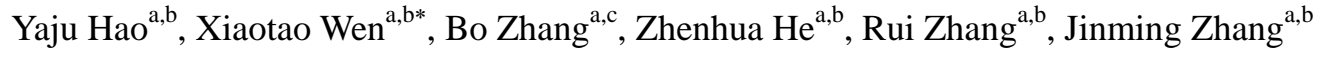 \\ ${ }^{\text {a }}$ State Key Laboratory of Oil \& Gas Reservoir Geology and Exploitation, Chengdu University of Technology, Chengdu, 610059, China \\ ${ }^{\mathrm{b}}$ College of Geophysics, Chengdu University of Technology, Chengdu, 610059, China \\ ${ }^{c}$ The University of Alabama, Department of Geological Sciences, Tuscaloosa, AL, 35487, USA
}

ABSTRACT:

Quality factor, Q, is a parameter that characterizes the energy dissipation during seismic wave propagation. The reservoir pore is one of the main factors that affect the value of Q. Especially, when pore space is filled with oil or gas, the rock usually exhibits a relative low Q value. Such a low $\mathrm{Q}$ value has been used as a direct hydrocarbon indicator by many researchers. The conventional Q estimation method based on spectral ratio suffers from the problem of waveform tuning; hence, many researchers have introduced time-frequency analysis techniques to tackle this problem. Unfortunately, the window functions adopted in time-frequency analysis algorithms such as continuous wavelet transform (CWT) and S-transform (ST) contaminate the amplitude spectra because the seismic signal is multiplied by the window functions during time-frequency decomposition. The basic assumption of the spectral ratio method is that there is a linear relationship between natural logarithmic spectral ratio and frequency. However, this assumption does not hold if we take the influence of window functions into consideration. In this paper, we first employ a recently developed two-parameter generalized S-transform (GST) to obtain the time-frequency spectra of seismic traces. We then deduce the non-linear relationship between natural logarithmic spectral ratio and frequency. Finally, we obtain a linear relationship between natural logarithmic spectral ratio and a newly defined parameter $\gamma$ by ignoring the negligible second order term. The gradient of this linear relationship is 1/Q. Here, the parameter $\gamma$ is a function of frequency and source wavelet. Numerical examples for VSP and post-stack reflection data confirm that our algorithm is capable of yielding accurate results. The Q-value results estimated from field data acquired in western China show reasonable comparison with oil-producing well location.

Keywords: Q factor estimation, Attenuation, Spectral-ratio method, Generalized S-transform

\section{Introduction}

\footnotetext{
${ }^{*}$ Corresponding author at: College of Geophysics, Chengdu University of Technology, \#1 Erxianqiao Dongsan Road, Chengdu, Sichuan 610059, China.

E-mail address: 383833129 @qq.com; wenxiaotao@cdut.cn.
} 
rock physical properties such as lithology, porosity, and permeability, just to mention a few (Johnston et al., 1979; Winkler and Nur, 1982; Rubino et al., 2012; Chabyshova and Goloshubin, 2014). However, one of the most interesting factors published by researchers is the fluid type within reservoir pore (Winkler and Nur, 1982; Johnson, 2011; Chabyshova and Goloshubin, 2014). It has been found that Q-value is lower in gas-bearing rocks than in those saturated with oil and water (Johnston et al., 1979; Klimentos, 1995). Nur et al. (1984) observed larger amplitude loss in oil saturated rocks than in those saturated with brine. Similarly, Behura et al. (2007) observed very low shear-wave Q-value (i.e., in a large temperature range, $Q_{s}<10$ ) in heavy-oil saturated sandstone. In this case, Q-value should be used as a direct hydrocarbon indicator, to some extent.

The spectral ratio technique is the most commonly used method for $\mathrm{Q}$ factor estimation. This algorithm is based on the assumption that the natural logarithmic spectral ratio is a linear function of frequency (Futterman, 1962; Tonn, 1991). The gradient of this linear relationship is 1/Q. To calculate the spectral ratio, we have to obtain the amplitude spectra of the reflected waves from the top and bottom of the reservoir. Unfortunately, in practice, field seismic reflections are always interfering with each other, to some extent. Therefore, it is inappropriate to compute $\mathrm{Q}$ factors by directly comparing the amplitude spectra in the interfering case (Wang, 2014). Reine et al. (2009) adopted time-frequency transform techniques including STFT, Gabor transform (GT), ST and CWT to yield interfering waveforms' amplitude spectra. They then used these amplitude spectra to invert Q-value and concluded that $\mathrm{Q}$ inversion methods based on variable-window time-frequency transforms (i.e., ST and CWT) are more robust than fixed-window time-frequency transform (i.e., STFT and GT) based methods. Similarly, many other researchers have also employed time-frequency analysis techniques to acquire the amplitude spectra of tuning seismic signal during the process of Q estimation (Wang, 2004; Li and Liu, 2015). It has been found, however, that Q estimation methods based on time-frequency analysis are highly affected by the window functions. Zhao and Ge (2008) deduced the equations for Q factor inversion based on CWT. They verified that if we ignore the wavelet base function used on CWT, inaccurate results will be obtained during Q inversion. Moreover, Wang et al. (2015) investigated different consequences caused by different window functions in the process of $\mathrm{Q}$ estimation.

In this paper, we also use time-frequency analysis technique to tackle the tuning effect. The time-frequency transform algorithm adopted in this paper is a two-parameter generalized $\mathrm{S}$ transform (GST) that was proposed by Chen et al. (2009a, 2009b). We can adjust the shape of window function by changing two parameters $(\lambda$ and $p$ ) to adapt to different non-stationary seismic signals (Chen et al., 2009a, 2013; Zhou et al., 2014). This trait can be considered to be superior to ST and CWT. To eliminate the influence of the window function employed in GST, we further derive $\mathrm{Q}$ factor inversion equations by combining spectral-ratio algorithm and GST. We 
find that there exists a quadratic relationship between the inverse of $Q$, and the natural logarithmic spectral ratio. Such a quadratic relationship is another evidence that proves that it is inadequate to employ spectral ratio technique directly after preforming time-frequency analysis on seismic signals. In addition, obtaining the parameters for the source wavelet is a prerequisite for the

\section{Foundations of Q Estimation Based on GST}

Suppose that the Fourier transform of a source wavelet is a Gaussian Function (Quan and Harris, 1997; Zhao and Ge., 2008; Hu et al., 2011), which can be expressed as $S_{0}(f)=e^{-\left(2 \pi f-2 \pi f_{d}\right)^{2} / m}$, where $f_{d}$ is the dominant frequency, and $m$ is a scalar controlling the frequency bandwidth (The bandwidth increases with $m$ ). According to dissipation theory (Futterman, 1962), after propagation time $t^{*}$, the wavelet in frequency domain can be expressed as (Gazdag and Sguazzero, 1984)

$S(f)=P \cdot e^{-\left(2 \pi f-2 \pi f_{d}\right)^{2} / m} e^{-i 2 \pi f t^{*}} e^{-\pi f t^{*} / Q}$,

where $Q$ is the quality factor and $P$ is the energy loss due to geometry spreading and energy partitioning.

Chen et al. (2009a, 2009b) proposed a two-parameter generalized S transform (GST) that can be expressed as

$\operatorname{GST}(\tau, f)=\int_{-\infty}^{+\infty}\left[S\left(f+f_{a}\right) e^{-\frac{2 \pi^{2} f_{a}^{2}}{\lambda^{2} f^{2 p}}}\right] e^{i 2 \pi f_{a} \tau} d f_{a} \quad(\lambda, p>0)$,

where $S\left(f_{a}\right)$ is the Fourier transform of seismic signal $s(t), \tau$ is time, and $\lambda$ and $p$ are the scaling parameters that together control the Gaussian window function.

Denote

$W\left(f_{a}\right)=e^{-\frac{2 \pi^{2} f_{a}^{2}}{\lambda^{2} f^{2 p}}}$,

which is the Fourier transform of Gaussian window function. We can adjust its shape to accommodate different non-stationary signals by changing the two scaling parameters ( $\lambda$ and $p$ ) conveniently, which is an advantage over STFT, GT, CWT, and ST (Chen et al., 2009b; Chen et al., 2013).

Substituting equation 2 with equation 1 , we can get 
$\operatorname{GST}(\tau, f)=\int_{-\infty}^{+\infty}\left[P \cdot e^{-\left[2 \pi\left(f+f_{a}\right)-2 \pi f_{d}\right]^{2} / m} e^{-i 2 \pi\left(f+f_{a}\right) t^{*}} e^{-\pi\left(f+f_{a}\right) t^{*} / Q} e^{-\frac{2 \pi^{2} f_{a}^{2}}{\lambda^{2} f^{2 p}}}\right] e^{i 2 \pi f_{a} \tau} d f_{a}$.

We then obtain the following equation (see the detail deduction procedure in the appendix):

$G S T(\tau, f)=P \cdot \sqrt{\frac{1}{\frac{4 \pi}{m}+\frac{2 \pi}{\lambda^{2} f^{2 p}}}} e^{-\left[\frac{\left(2 \pi f-2 \pi f_{d}\right)^{2}}{m}+\frac{\pi f t^{*}}{Q}\right]+\frac{\varsigma^{2}-\left(t^{*}-\tau\right)^{2}}{\frac{4}{m}+\frac{2}{\lambda^{2} f^{2 p}}}} e^{2 i\left[\varsigma\left(t^{*}-\tau\right)-\pi f t^{*}\right]}$,

where $\varsigma=\frac{4 \pi f}{m}-\frac{4 \pi f_{d}}{m}+\frac{t^{*}}{2 Q}$.

Equation 5 gives the time-frequency spectrum of the attenuated seismic wavelet. The amplitude spectrum can be expressed as

$|G S T(\tau, f)|=P \cdot \sqrt{\frac{1}{\frac{4 \pi}{m}+\frac{2 \pi}{\lambda^{2} f^{2 p}}}} e^{-\left[\frac{\left(2 \pi f-2 \pi f_{d}\right)^{2}}{m}+\frac{\pi f t^{*}}{Q}\right]+\frac{\varsigma^{2}-\left(t^{*}-\tau\right)^{2}}{\frac{4}{m}+\frac{2}{\lambda^{2} f^{2 p}}}}$.

If the time parameter $\tau$ is set to $t^{*}$, equation 6 can be written as

$\left|G S T\left(t^{*}, f\right)\right|=P \cdot \sqrt{\frac{1}{\frac{4 \pi}{m}+\frac{2 \pi}{\lambda^{2} f^{2 p}}}} e^{-\left[\frac{\left(2 \pi f-2 \pi f_{d}\right)^{2}}{m}+\frac{\pi f t^{*}}{Q}\right]+\frac{\left(\frac{4 \pi f}{m}-\frac{4 \pi f f_{d}}{m}+\frac{t^{*}}{2 Q}\right)^{2}}{\frac{4}{m}+\frac{2}{\lambda^{2} f^{2 p}}}}$.

In equation 6 , the partial derivative $|G S T(\tau, f)|_{\tau}$ of the amplitude spectrum $|G S T(\tau, f)|$ can be written as

$|G S T(\tau, f)|_{\tau}=\frac{\partial|G S T(\tau, f)|}{\partial \tau}=|G S T(\tau, f)| \cdot \frac{2\left(t^{*}-\tau\right)}{\frac{4}{m}+\frac{2}{\lambda^{2} f^{2 p}}}$.

105 If we set $|\operatorname{GST}(\tau, f)|_{\tau}$ to 0 , we obtain

$\tau=t^{*}$.

Equation 9 indicates that the amplitude spectrum at time $t^{*}$ corresponds to local maximum for each frequency components on the time-frequency map, which is also demonstrated by numerical experiments (Cai et al., 2015).

To obtain the $\mathrm{Q}$ factor between two reflections whose travel times are $t_{1}^{*}$ and $t_{2}^{*}$, respectively, we apply the spectral ratio method at time $t_{1}^{*}$ and $t_{2}^{*}$. We then obtain

$\ln \frac{\left|G S T\left(t_{2}^{*}, f\right)\right|}{\left|G S T\left(t_{1}^{*}, f\right)\right|}=-\pi f \frac{\Delta t}{Q}+\frac{\frac{4 \pi f}{m}-\frac{4 \pi f_{d}}{m}}{\frac{4}{m}+\frac{2}{\lambda^{2} f^{2 p}}} \frac{\Delta t}{Q}+\frac{1}{\frac{4}{m}+\frac{2}{\lambda^{2} f^{2 p}}} \frac{t_{2}^{* 2}-t_{1}^{* 2}}{4 Q^{2}}+\ln \frac{P_{2}}{P_{1}}$,

where $\Delta t=t_{2}^{*}-t_{1}^{*}, P_{1}$ and $P_{2}$ are the energy losses, which are independent of frequency at time $t_{1}^{*}$ and $t_{2}^{*}$, respectively. $\left|G S T\left(t_{1}^{*}, f\right)\right|$ and $\left|G S T\left(t_{2}^{*}, f\right)\right|$ are the amplitude spectra at time $t_{1}^{*}$ and $t_{2}^{*}$. Equation 10 can be further expressed as

$\hat{S}(f)=\gamma \cdot \frac{1}{Q}+\gamma^{\prime} \cdot \frac{1}{Q^{2}}+\hat{P}$,

where $\left\{\begin{array}{c}\gamma=-\pi f \Delta t+\frac{\frac{4 \pi f}{m}-\frac{4 \pi f}{m}}{\frac{4}{m}+\frac{2}{\lambda^{2} f^{2 p}}} \Delta t \\ \gamma^{\prime}=\frac{1}{\frac{4}{m}+\frac{2}{\lambda^{2} f^{2 p}}} \frac{t_{2}^{* 2}-t_{1}^{* 2}}{4}\end{array}, \hat{S}(f)=\ln \frac{\left|G S T\left(t_{2}^{*}, f\right)\right|}{\left|G S T\left(t_{1}^{*}, f\right)\right|}, \hat{P}=\ln \frac{P_{2}}{P_{1}}\right.$.

Equation 11 shows that there exists a quadratic relationship between natural logarithmic spectral 
ratio and the inverse of $\mathrm{Q}\left(\mathrm{Q}^{-1}\right)$. In equation $11, \gamma$ has the same order of magnitude as $\gamma^{\prime}$.

Additionally, considering that $\mathrm{Q}$ factor usually ranges from tens to hundreds, we conclude $\frac{1}{Q} \gg \frac{1}{Q^{2}}$. Thus the contribution of the second term in equation 11 is negligible during the inversion procedure. We therefore obtain the expression

$\hat{S}(f)=\gamma \cdot \frac{1}{Q}+\hat{P}$.

Using to equation 12 , we can determine the $\mathrm{Q}$ factor by linearly fitting $\hat{S}(f)$ and $\gamma$.

\section{Application on Synthetic cases}

\subsection{Q factor inversion for synthetic VSP seismic data}

Fig. 1(a) and (b) show the primary velocity $V_{p}$ and $Q$ models, respectively. The values of these parameters are obtained from Wang (2014). Fig. 1(c) shows the result of zero-offset synthetic VSP results by using the method proposed by Ganley (1981). In this figure, for simplicity, we show direct waves and primary reflections. The wavelet is a Gaussian wavelet with a dominant frequency of $40 \mathrm{~Hz}$ and scalar value of 10000. The geophones range from $50 \mathrm{~m}$ to $1900 \mathrm{~m}$ with an incremental size of $5 \mathrm{~m}$. The 1D viscoelastic equation employed during forward modeling is expressed as

$\frac{\partial^{2} U}{\partial t^{2}}=\frac{M}{\rho} \frac{\partial^{2} U}{\partial z^{2}}$

where $M$ is the complex elasticity modulus. $\mathrm{Q}$ is defined by the ratio between the imaginary and real parts of $M, Q=\operatorname{Im}(M) / \operatorname{Re}(M)$ (Ganley, 1981). The complex velocity, $c$, is defined as $c=V_{R e}+i V_{I m}=\sqrt{M / \rho}$. The interval velocity in Figure 1 (a) is the real part of complex velocity, c (Wang, 2014).
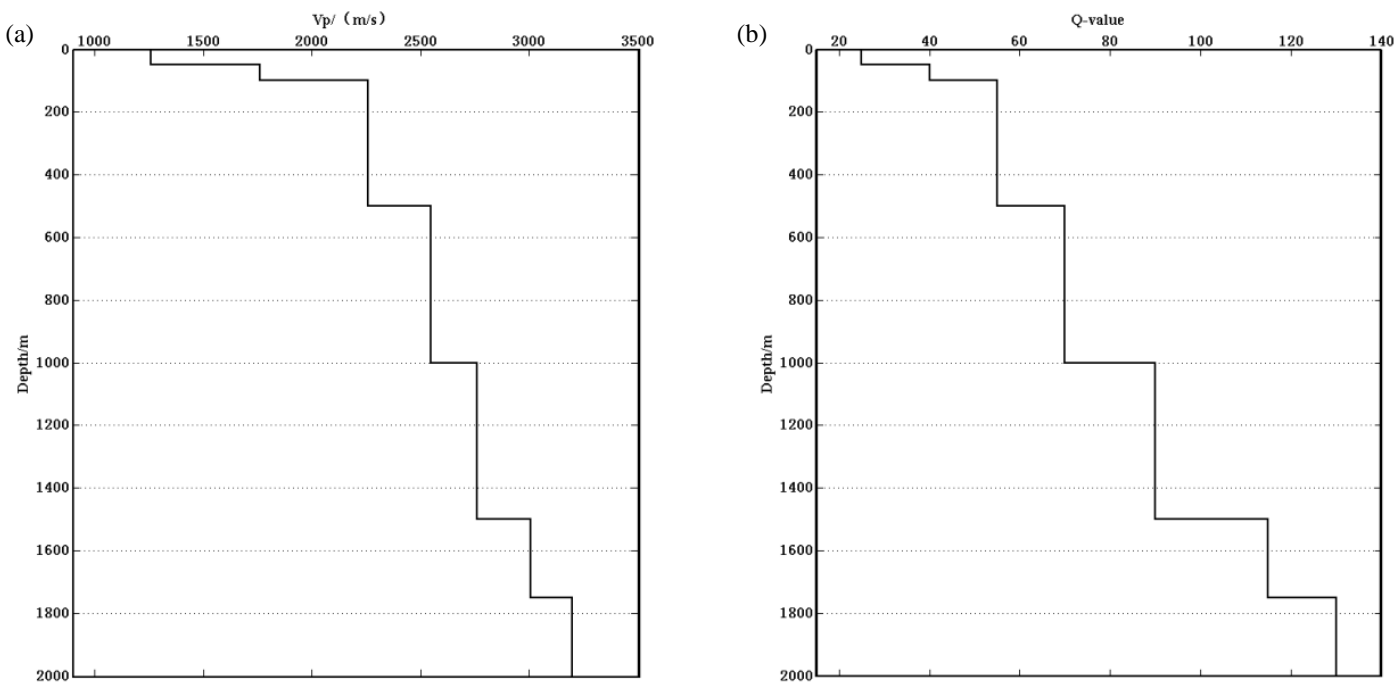


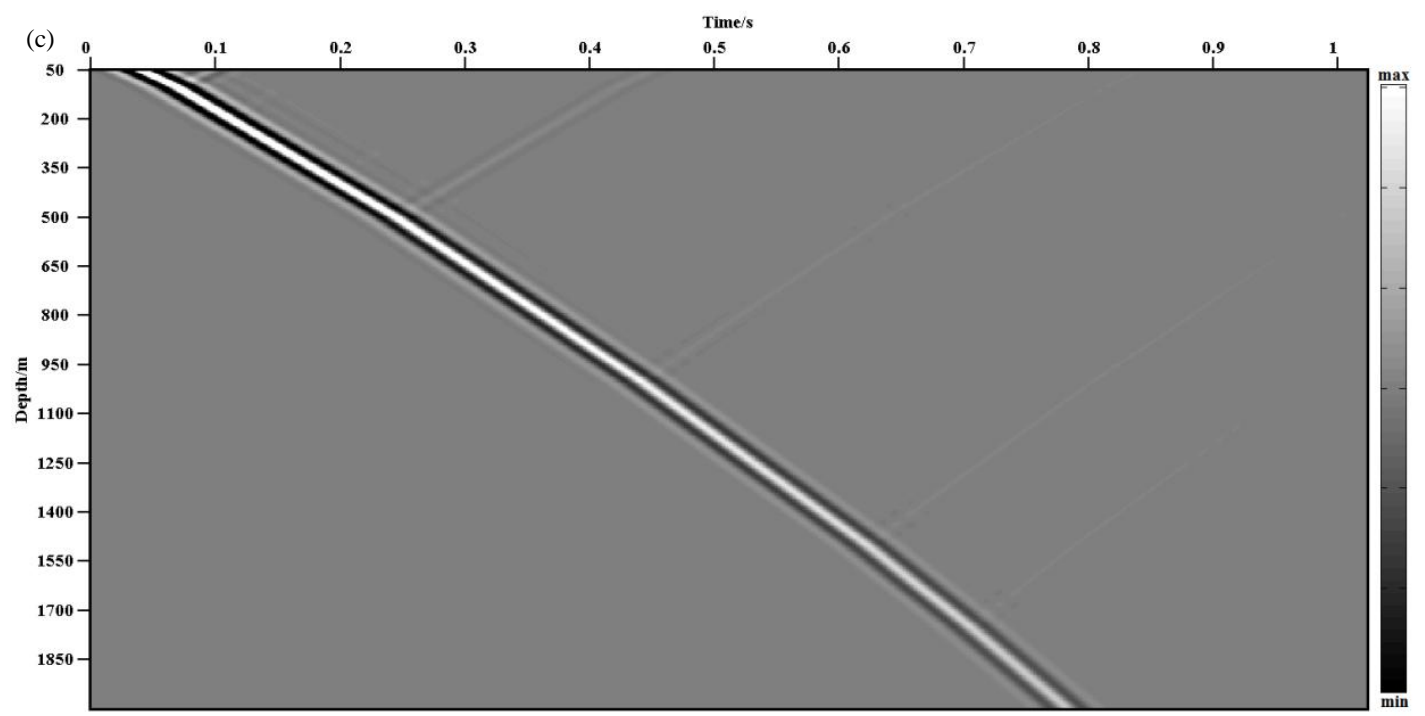

Fig.1. VSP modeling. (a) and (b) are the velocity and Q models, and (c) is the synthetic VSP data.
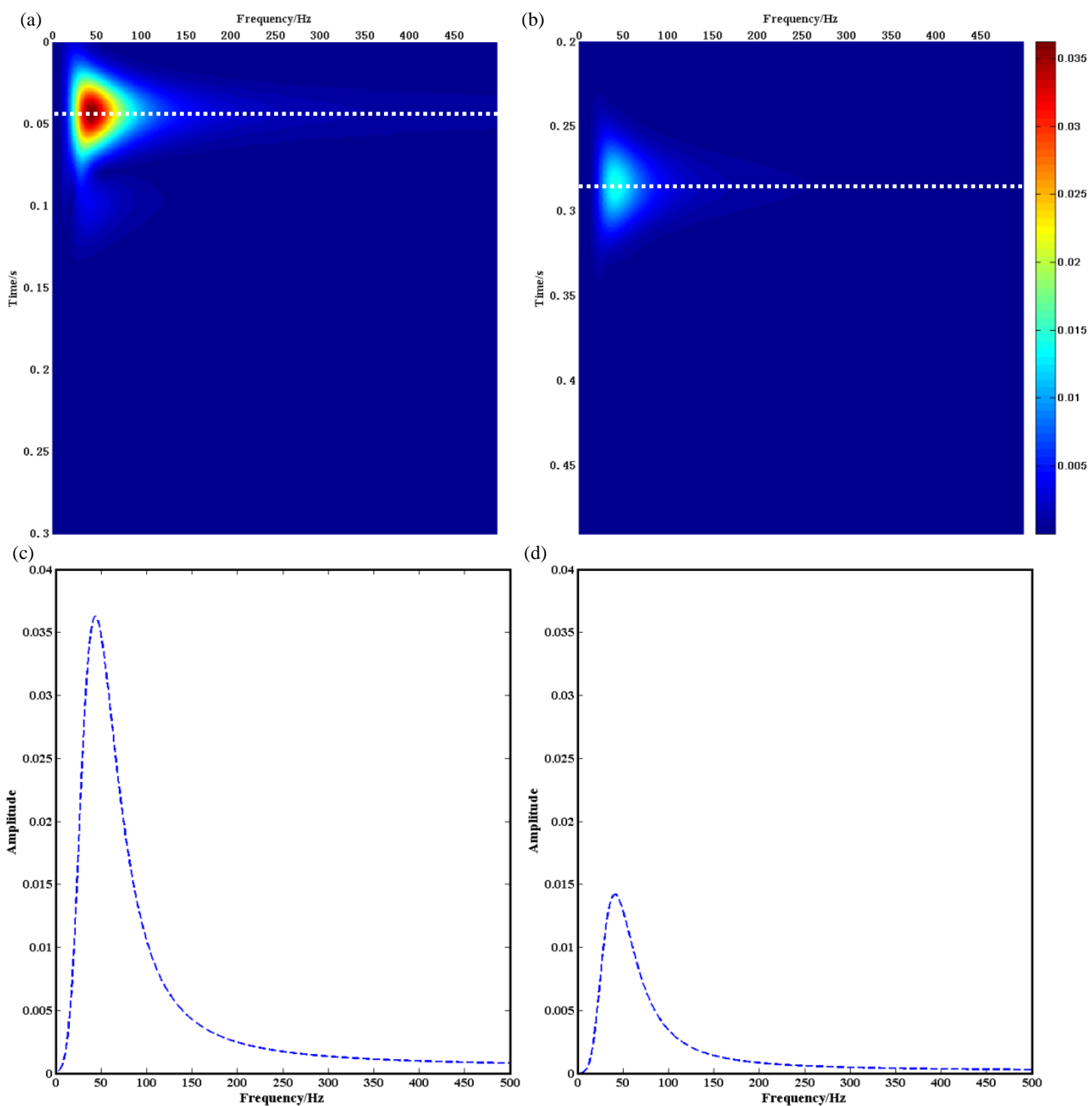

Fig. 2. (a) and (b) are GST time-frequency spectra corresponding to the first and $111^{\text {th }}$ trace in Fig. 1(c); (c) and (d) are amplitude spectra located by the white dashed lines in Fig. 2(a) and (b). 
Fig. 2(a) and (b) are time-frequency spectra (computed by GST) corresponding to the first and 111th traces in Fig. 1(c). Note that we have a notable energy loss for trace number 111 due to dissipation during propagation. The value of $\lambda$ and $p$ used for GST are 3 and 1 , respectively. In practice, we can determine the set of $\lambda$ and $p$ by several times of numerical experiments to get a reasonable result (Chen et al., 2009b). Fig. 2(c) and (d) are the amplitude spectra along the white dashed lines in Fig. 2(a) and (b), respectively. From the amplitude spectra, shown in Fig. 2(c) and (d), we can then calculate the natural logarithmic spectral ratio directly.

The cross-plot of natural logarithmic spectral ratio versus frequency is shown in Fig. 3(a). Clearly, it's a complicated nonlinear relationship. In the low frequency part, the relation of natural logarithmic spectral ratio verses frequency is approximately linear. We can obtain an approximation of the $\mathrm{Q}$ factor through linear regression. However, in the high frequency part, the curve gets severely distorted, caused by the window function used in GST. High frequency information plays a significant role in obtaining a high resolution inversion result; thus, we have to utilize the high frequency information accurately.

155 On the contrary, there exists a very good linear relationship between the logarithmic spectral ratio and the newly defined parameter $\gamma$ in our algorithm (shown in Fig. 3(b)). We can therefore compute the Q-values by linear regression according to Eq. (12). In this case, we can simultaneously use the low and high frequency parts information. It is certainly a good method for improving the resolution of $\mathrm{Q}$ estimation. Furthermore, we use the spectrum obtained from the first trace as the reference spectrum and apply Eq. 12 to obtain the equivalent $Q$ factors between the first trace and all other traces. The blue solid and red dashed lines in Fig. 4 are the theoretical and inverted equivalent $\mathrm{Q}$ values. If two adjacent traces' spectra are respectively treated as a reference and measured spectra, we can obtain the Q-value of every depth point.
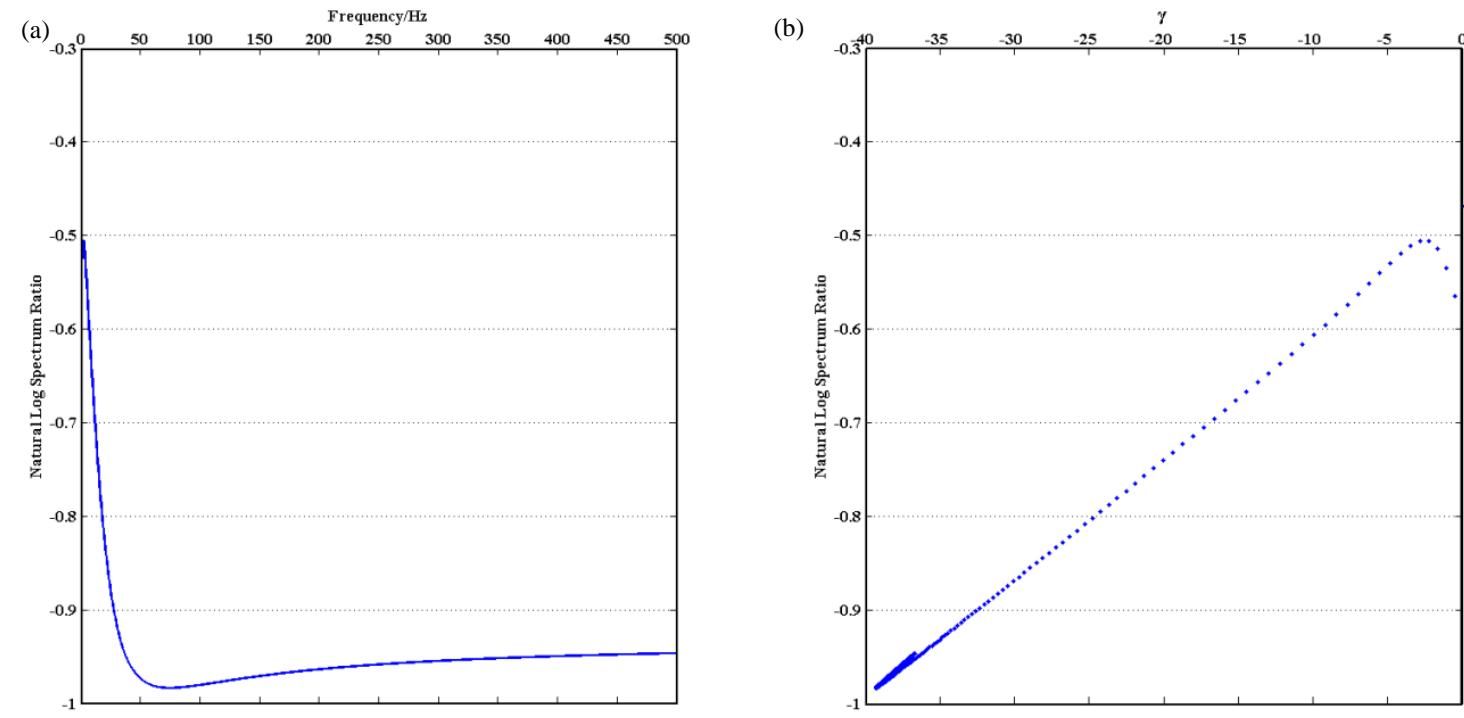

Fig. 3. Natural logarithmic spectral ratio versus (a) frequency and (b) newly defined parameter $\gamma$. 

respectively. The perfect match between theoretical and inverted values proves the effectiveness of our method.

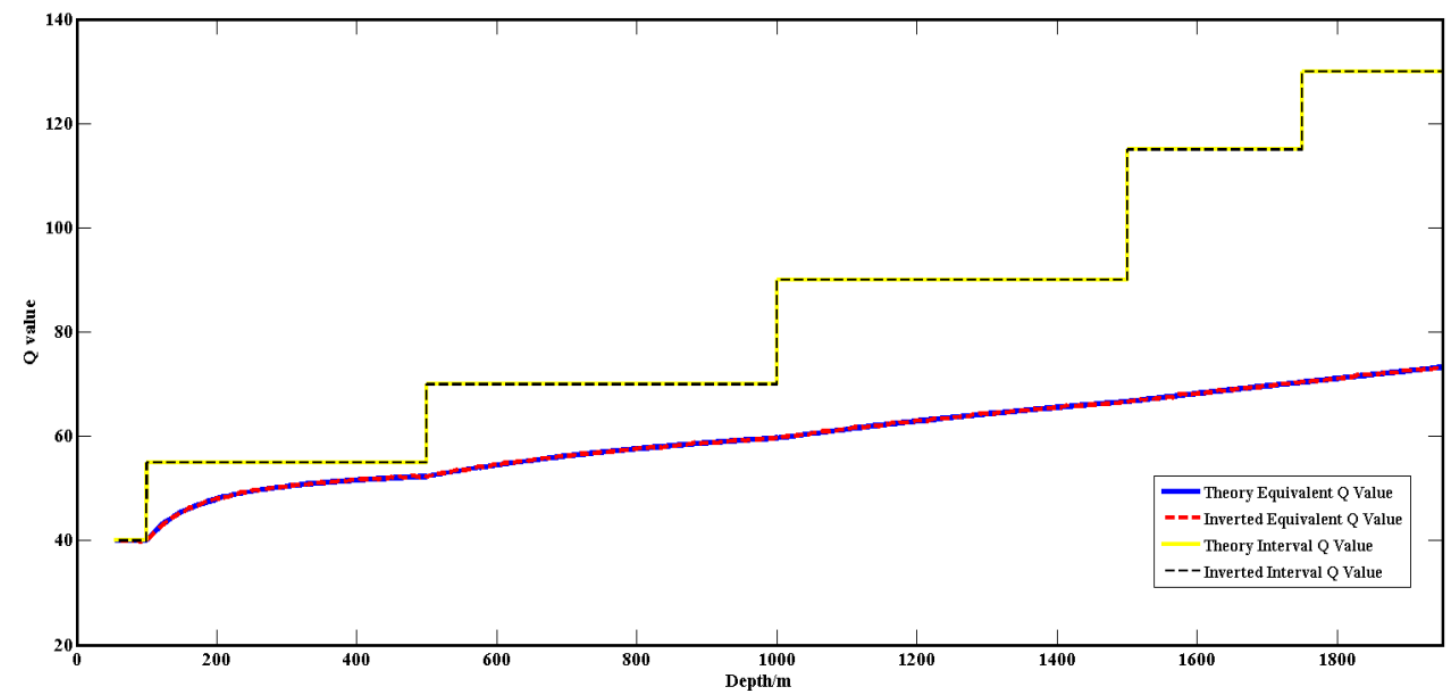

Fig. 4. Q-values obtained by our algorithm. Solid lines represent theoretical values and dashed lines represent inverted values.

\subsection{Q factor inversion for synthetic poststack seismic data}

We also demonstrate the effectiveness of our algorithm on synthetic poststack seismic data by considering the popularity of reflection seismology in oil and gas exploration. The model contains an anticline that represents a hydrocarbon reservoir with a low $\mathrm{Q}$ value of 15 (circled by dashed rectangle in Fig. 5(a)). We first generate a poststack seismic profile (Fig. 5(b)) by using Eq. 13. The wavelet is a Gaussian wavelet with dominant frequency $\left(f_{d}\right)$ of $40 \mathrm{~Hz}$ and scaling value $(m)$ of 10000. In Fig. 5(c), the left panel shows the time-frequency spectrum of the $52^{\text {th }}$ trace in Fig. 5(b) $(\mathrm{X}=510 \mathrm{~m})$, and the right panel shows the equivalent $\mathrm{Q}$ value trace obtained from this time-frequency spectrum. When we calculate the equivalent $Q$ value trace in Fig. 5(c) using Eq. 12, the amplitude spectrum at every time index on time-frequency the spectrum map is set as a measured spectrum. In this case, we can obtain an inversion value at every time index. Nonetheless, only those inversion values marked by red stars in Fig. 5(c) are correct because Eq. 12 is correct only at those time indexes where the time-frequency spectra is located at a local maximum value (see Eq. 7). Fig. 5(d) shows the inverted equivalent Q-value profile with a good resolution at the gas reservoir. Similarly, in Fig. 5(e), the left panel shows time-frequency spectrum of the $52^{\text {th }}$ trace in Figure $5 \mathrm{~b}(X=510 \mathrm{~m})$, and the right panel shows an inverted interval Q-value trace. When we calculate the interval Q value in Fig. 5(e), we first pick the local maximum value (indicated by the brown dotted line) on the time-frequency spectrum map. We then set two adjacent spectra as reference spectrum and measured spectrum, respectively, to 
calculate $\hat{S}(f)$. Finally, we obtain the interval Q value by linearly fitting $\hat{S}(f)$ and $\gamma$. Interval Q model can be obtained by performing this procedure trace by trace (Fig. 5(f)). The incorrect $Q$ value, in inverted Q model, is due to the heavy tuning effect at the marge of the reservoir.
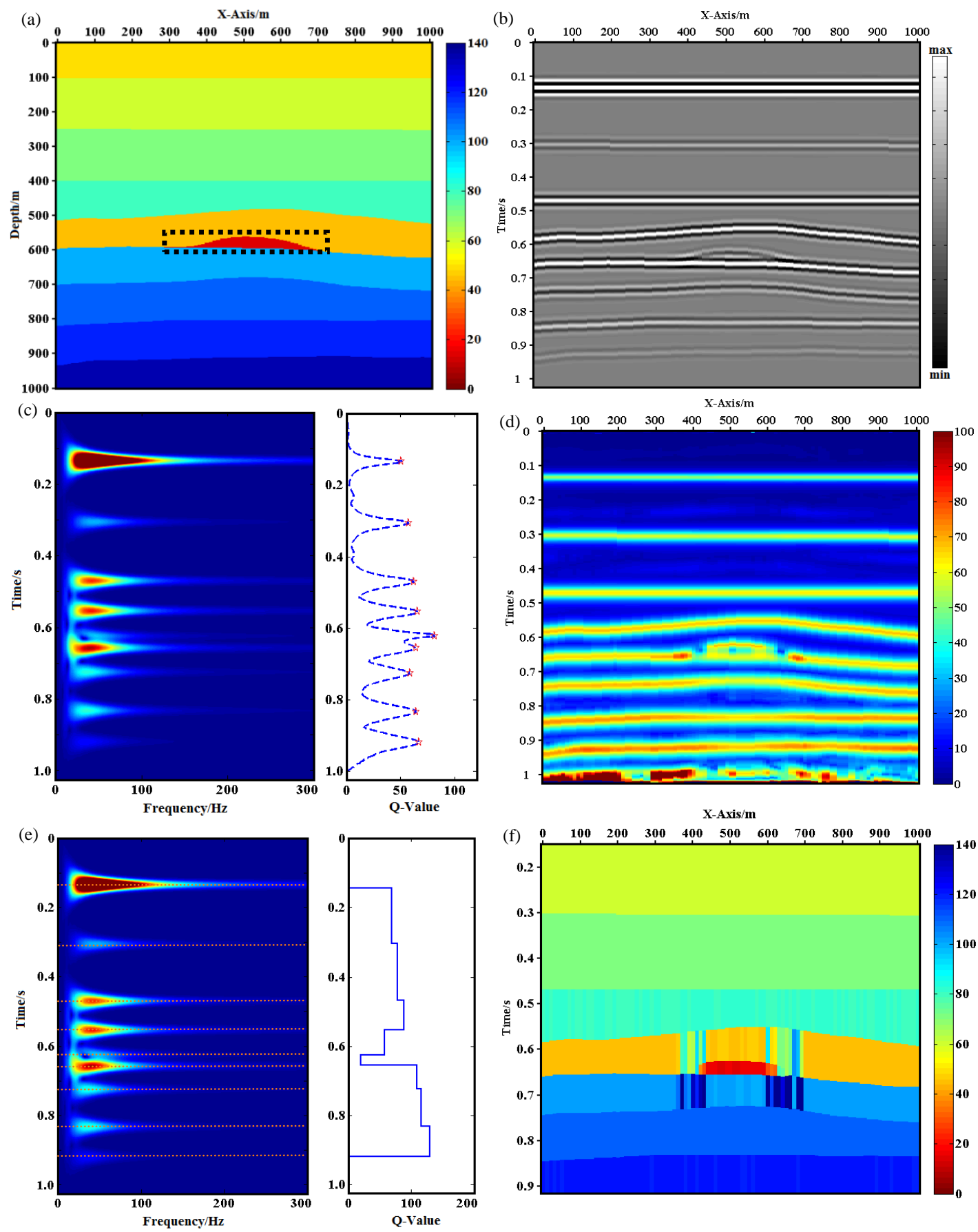

Fig. 5. (a) True Q model; (b) synthetic poststack seismic section; (c) time-frequency spectrum and inverted equivalent $\mathrm{Q}$ trace; (d) inverted equivalent $\mathrm{Q}$ profile; (e) time-frequency spectrum and inverted interval Q trace; (f) inverted interval Q model.

We use the Gaussian wavelet during the forward modeling for the above case. There are two parameters $\left(f_{d}\right.$ and $\left.m\right)$ used to determine a Gaussian wavelet. However, in practice, the source wavelet's spectrum does not strictly follow Gaussian distribution (Rickett, 2006; Hu et al., 2011). 
It is necessary to test the efficiency of our algorithm when the source wavelet's Fourier spectrum is a non-Gaussian function. We employ Ricker wavelet to generate a synthetic poststack record (Fig. 6(a)), considering that it is a commonly used wavelet in forward and inversion methods. Here, the dominant frequency of Ricker wavelet is $40 \mathrm{~Hz}$. This Ricker wavelet is plotted as a time signal and amplitude spectrum in Fig. 6(b) (red dashed curves). We first simulate the best fitting two-parameter Gaussian wavelet by least-square regression strategy. The dominant frequency $\left(f_{d}\right)$ and scaling value $(m)$ of simulated Gaussian wavelet are $40 \mathrm{~Hz}$ and 27000 , respectively. We also plot this simulated wavelet as a time signal and amplitude spectrum in Fig. 6(b) (blue dashed curves). Note that there exists a negligibly subtle difference between the simulated and original Ricker wavelet in both the time and frequency domain. We then obtain the equivalent and interval Q profile by repeating the procedure above. Figures 6(c) are 6(d) show the inverted equivalent and interval Q factor values, respectively. Note that we obtain almost the same inverted results for the Gaussian wavelet case as the Ricker wavelet case. Here, we note that, in practice, the source wavelet's Fourier spectrum can be simulated by the Gaussian function especially for land seismic exploration (Lu and Wang, 2011).
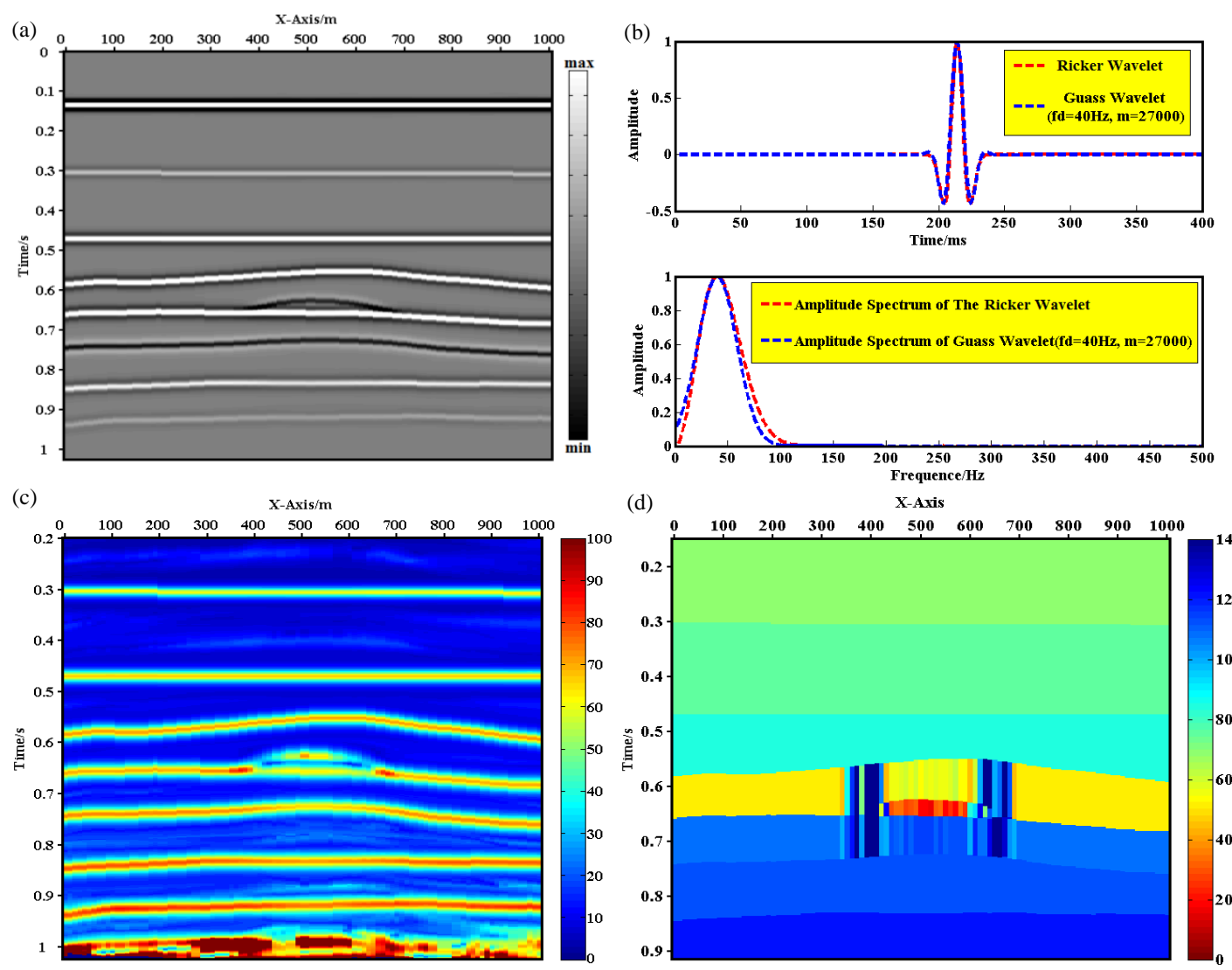

(d)

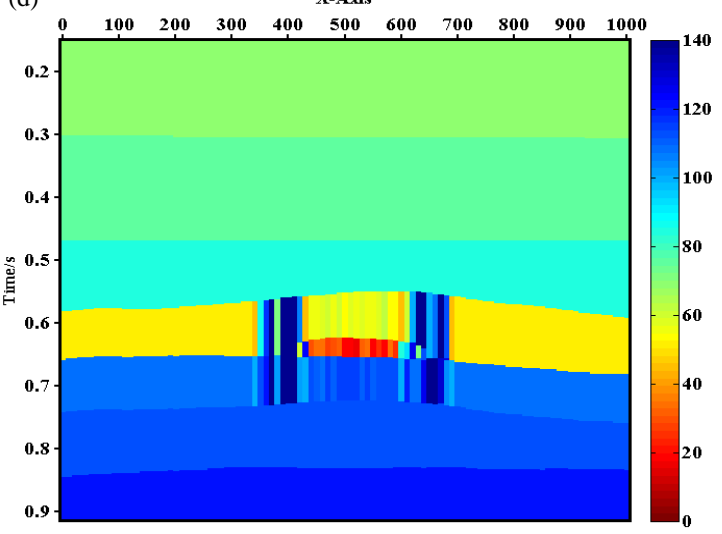

Fig. 6. (a) Synthetic poststack seismic data yield by using a Ricker wavelet; (b) least-square fitting the Ricker wavelet by using Gaussian wavelet; (c) inverted equivalent Q profile; (d) inverted interval Q model.

Figure 7 summarizes the workflow of $\mathrm{Q}$ factor inversion in this paper. The input data of our workflow is poststack seismic data and the output is the corresponding Q models. The workflow 
begins with noise suppression. We then simulate a two-parameter Gaussian wavelet by least-square fitting the source wavelet to obtain $f_{d}$ and $m$. To obtain the source wavelet, we employ methods that have been published (Yu et al., 2011; Kim et al., 2013). Alternatively, we could also use a statistical wavelet estimated from the seismic above the reservoir instead of the source wavelet, which is similar to the strategy adopted by Chai et al. (2016). Next we obtain time-frequency spectrum of every seismic trace by using GST. Finally, we apply equation 12 to obtain the Q model corresponding to the seismic data.

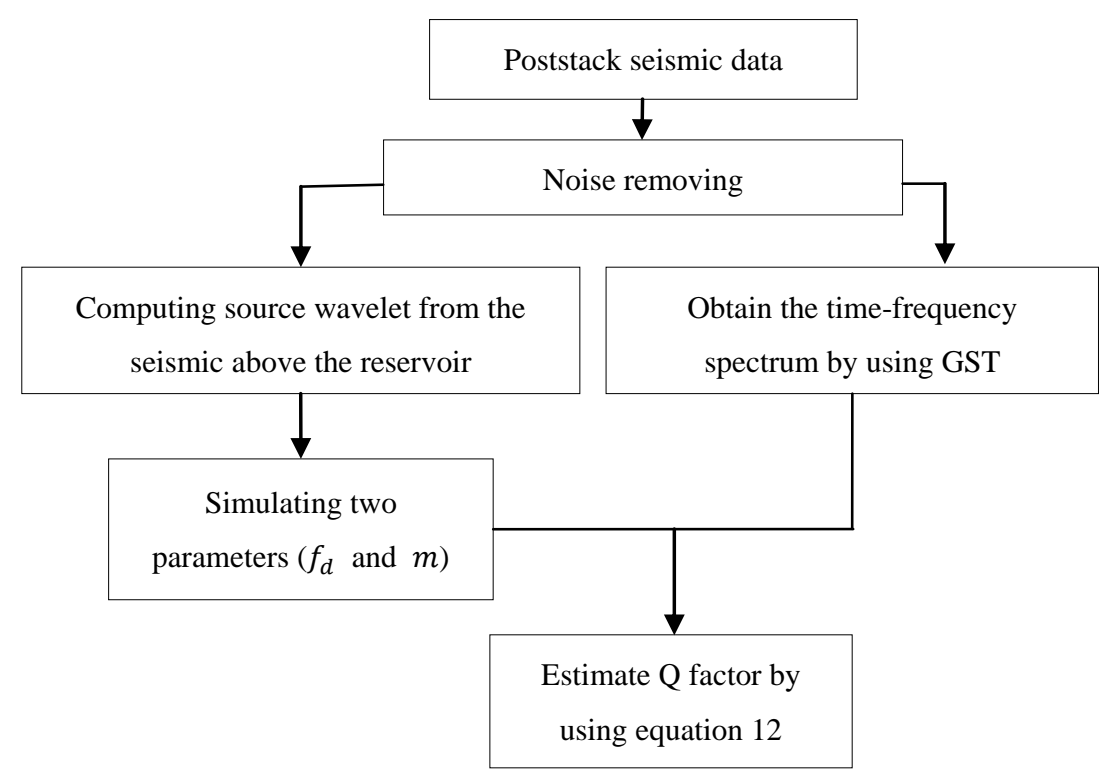

Fig. 7. Flow chart of $\mathrm{Q}$ inversion method in this paper.

\section{Applications to field data set}

To further demonstrate the capacity of our methods, we apply the workflow shown in Fig. 7 to a seismic data set acquired from West China. The data were acquired with 25-m shot spacing and 25-m receiver spacing. A raw section passing through Well-1 is shown in Fig. 8. The dashed magenta curve in Fig. 8 indicates the top of the target reservoir. The target sand reservoir (indicated by green dash line box in Fig. 8) is in Triassic age and the deposition environment is braiding river-deltas. Well-1 is one of the high production oil wells in this field. In Fig. 9 the seismic well tie is plotted with Gamma Ray, P-wave velocity, deep (red curve) and shallow (blue curve) laterolog resistivity measurements (LLD and LLS). The P-wave velocity exhibits relative low value at the reservoir (marked by red color zone) and there exists a positive difference between the deep and shallow resistivity logs, which demonstrate that the reservoir is hydrocarbon-bearing. We choose the reflection of a sandstone/shale interface indicated by the red double arrow both in Fig. 8 and Fig. 9 as our reference reflection. We then obtain the statistical 
wavelet from this early part of the traces. The estimated wavelet is located at the upper right in Fig. 8 and is almost a zero-phase wavelet. Given that we do not have additional information about the source signal and the more early part of the seismic traces, we can only treat this extracted wavelet as source wavelet and simulate the optimal Gaussian function to obtain $f_{d}$ and $m$. It will indeed induce error, but this error is moderate in this case, according our following analysis. The dominant frequency $\left(f_{d}\right)$ and scalar value $(m)$ of corresponding Gaussian wavelet are $54 \mathrm{~Hz}$ and 65000 , respectively.

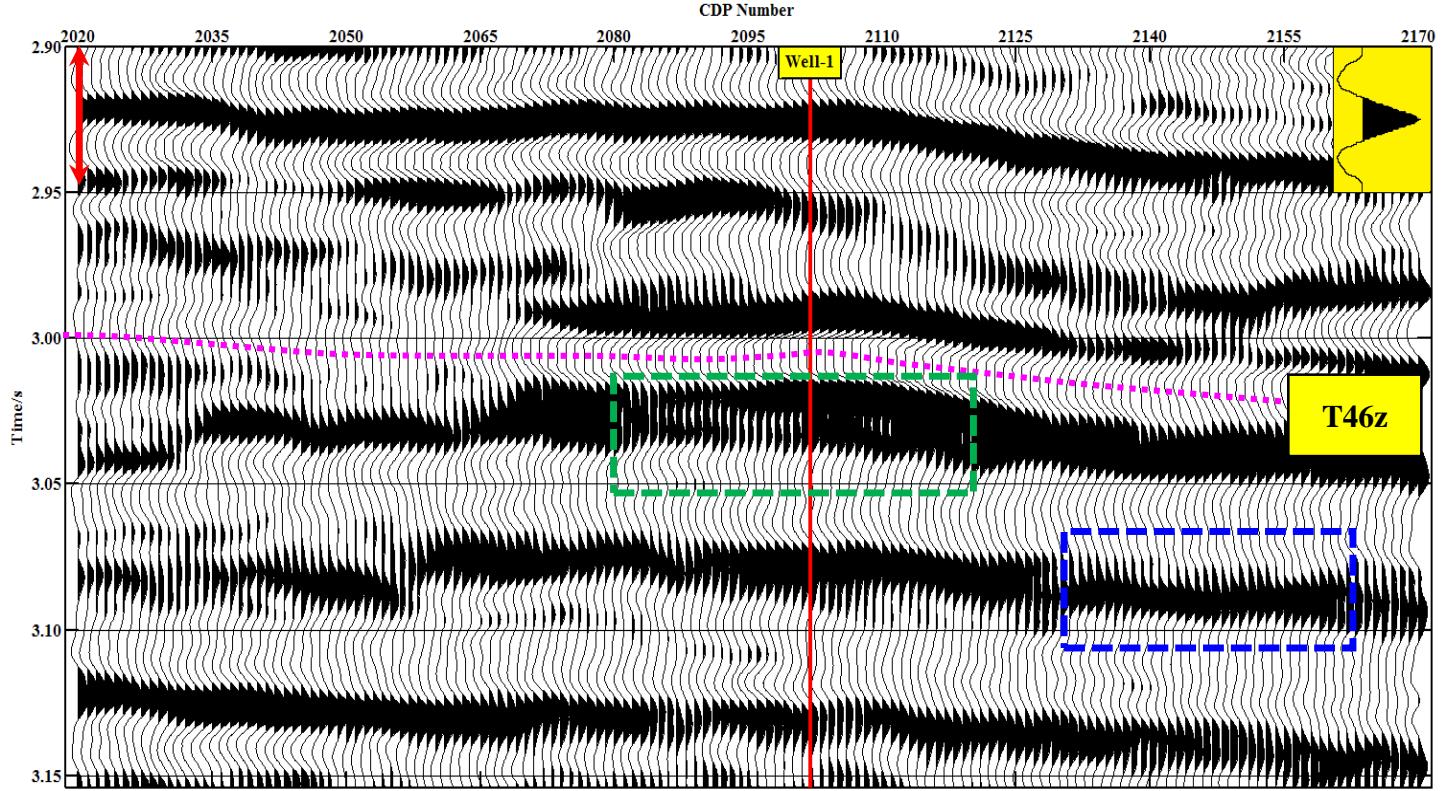

Fig. 8. Stacked seismic section passing through Well-1. The sand reservoir is indicated by green arrows.

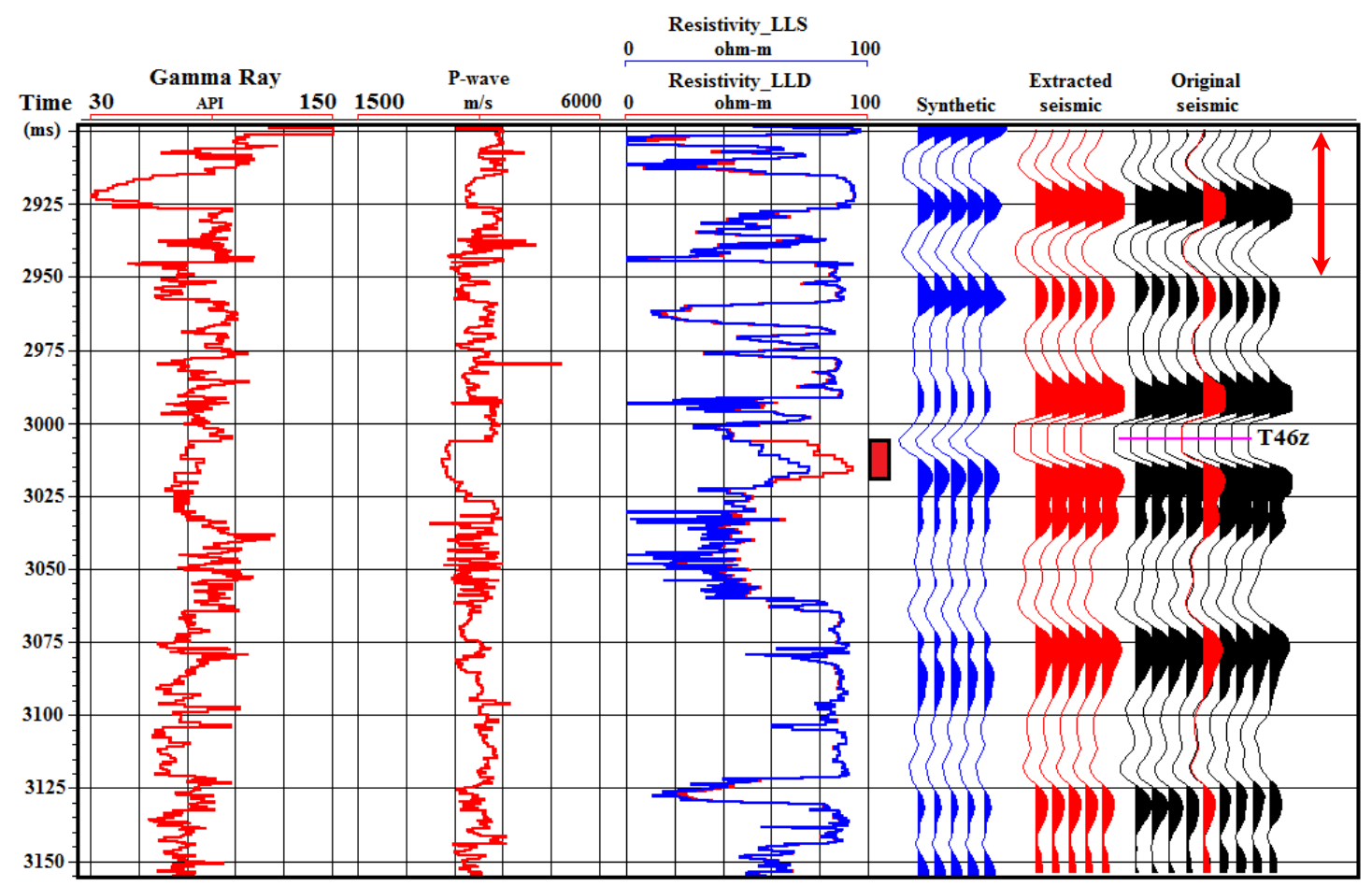

Fig. 9. The left three panels show well-log data of Gamma Ray, P-wave and Resistivity, respectively. The right-most panel shows the result of seismic-well tie. The red box indicates the location of oil reservoir. 

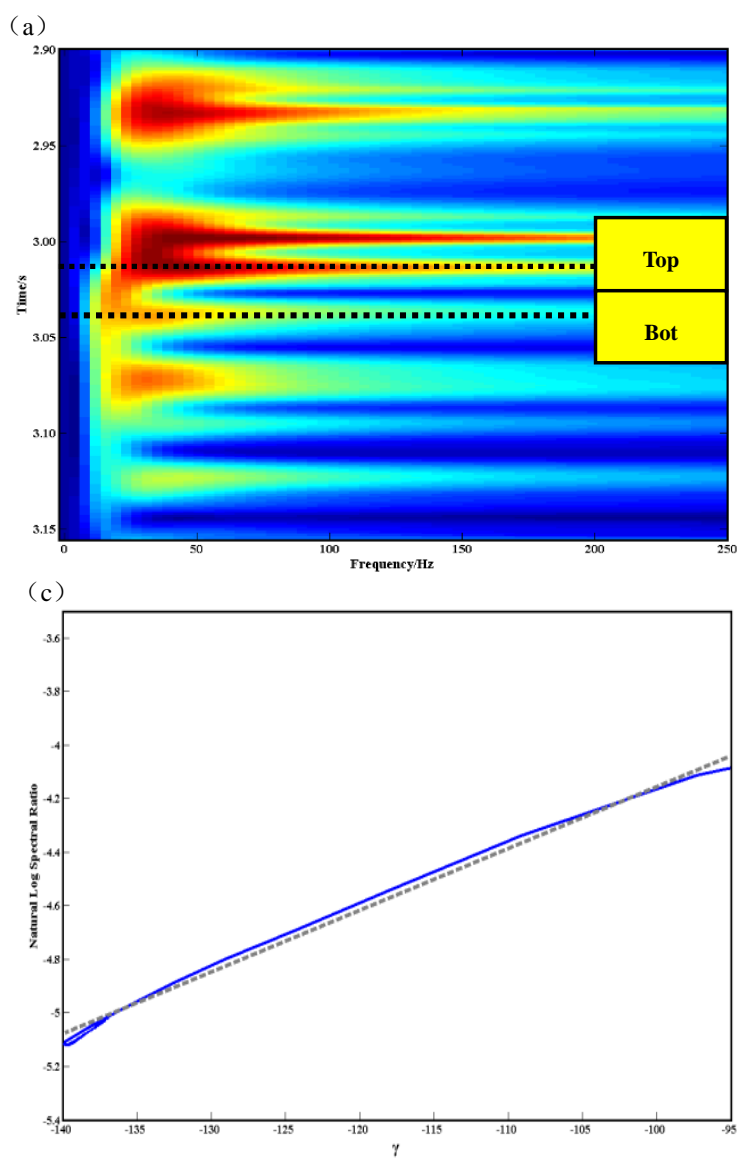

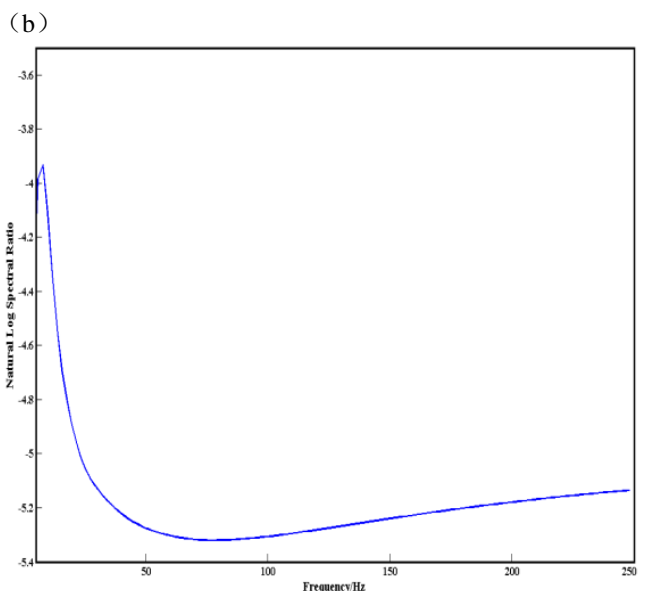

Fig. 10. (a) Time-frequency spectrum of the seismic trace nearby the borehole place; (b) crossplot of natural logarithm spectral ratio vs. frequency; (c) crossplot of natural logarithm spectral ratio vs. $\gamma$.

Fig. 10(a) illustrates the GST time-frequency spectrum of the seismic trace near the borehole (Well-1) location. The black dashed lines in Figure 10(a) indicate the top and bottom of the reservoir. Fig. 10(b) and (c) show the crossplot of natural logarithmic spectral ratio for the time index at the black dashed lines in Fig. 10(a) vs. frequency and $\gamma$, respectively. Note that the nonlinear relationship between the natural logarithmic spectral ratio and frequency and linear relationship between the natural logarithmic spectral ratio and $\gamma$, which demonstrates the efficiency of our algorithm. The gray dashed line in Fig. 10(c) is the simulated linear function between $\gamma$ and the natural logarithmic spectral ratio. We also note that although we use an extracted wavelet to alternate the true source signal, a linear relationship between natural logarithmic spectral ratio and $\gamma$ is obtained. This result testifies that the error caused by such approximation can be sustainable in this case.

Fig. 11(a) illustrates the inverted Q model corresponding to the seismic profile shown in Figure 8. We obtain anomalously low $\mathrm{Q}$ values around the borehole location at the reservoir zone. Fig. 11 (b) illustrates the $\mathrm{Q}$ profile which is obtained by traditional method (i.e. linear fitting natural logarithmic spectral ratio and frequency directly). The scope of $\mathrm{Q}$ abnormality is much larger than the result of our method. Because the lack of laboratory data about $\mathrm{Q}$ value, it's hard to evaluate which result is more reasonable. Fortunately, Q-value has a close relationship with frequency and we can qualitatively evaluate the magnitude of Q-value by the frequency components of reflection 
waves. Fig. 12 shows the amplitude spectra of reflection waves in the green and blue dashed line boxes in Fig. 8 (the corresponding location on Fig. 11(b) are indicated by green and blue arrows, respectively). It's clear that the frequency band of the waves in blue box is larger than that in green box. So, the absorptive capacity of the strata in green box is higher than that in blue box. We can conclude that the Q-value of the strata in green box is lower than that in blue box. The result obtained by our method (Fig. 11(a)) is consistent with the above conclusion, however, the result obtained by traditional method (Fig. 11(a)) do not conform to the above conclusion. In this case, our new method is more accurate than the traditional method.

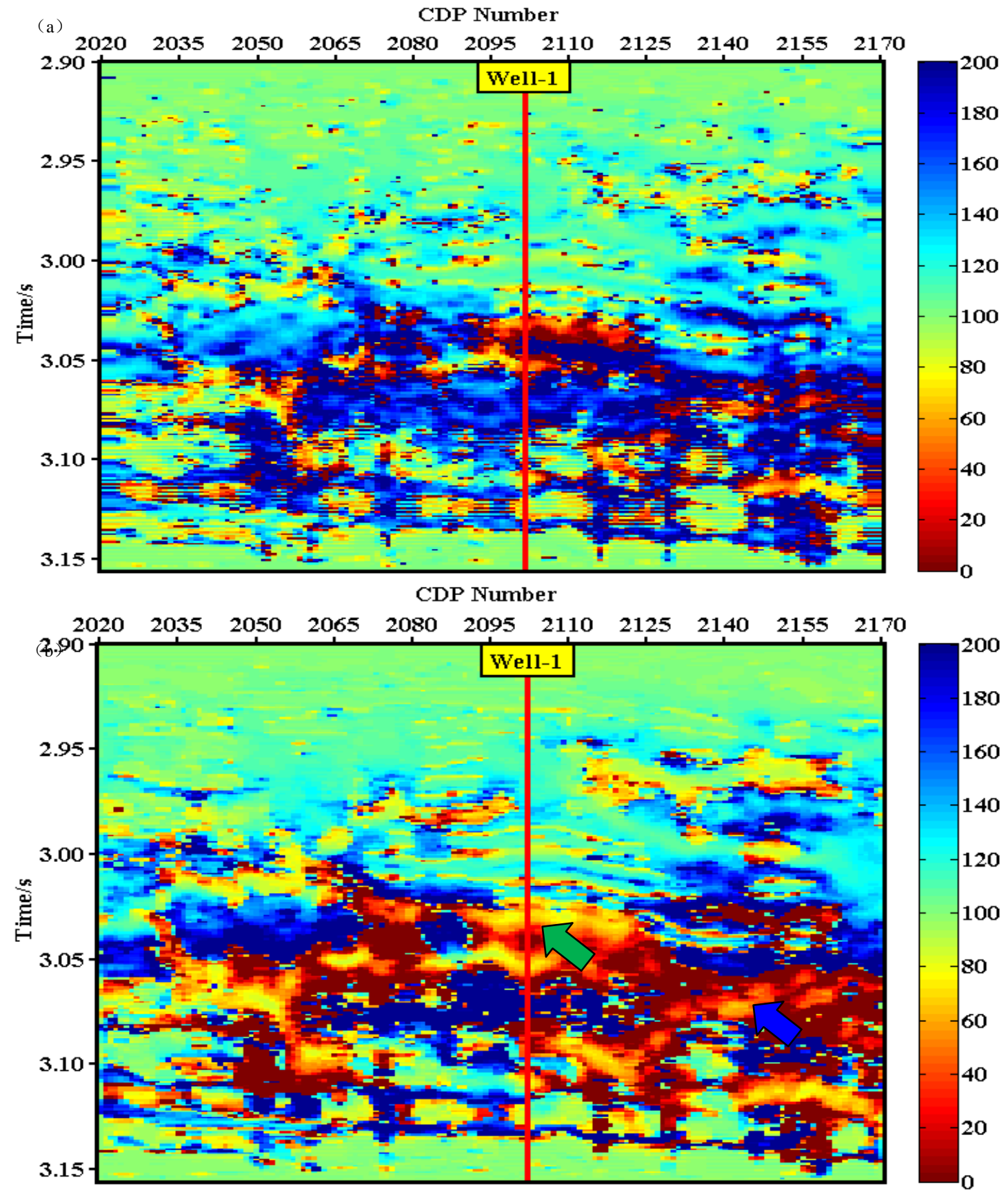

Fig. 11. Inverted Q model corresponding to the seismic profile shown in Figure 8. (a) shows the inverted Q section obtained by our method; (b) shows the inverted Q section obtain by traditional method. 


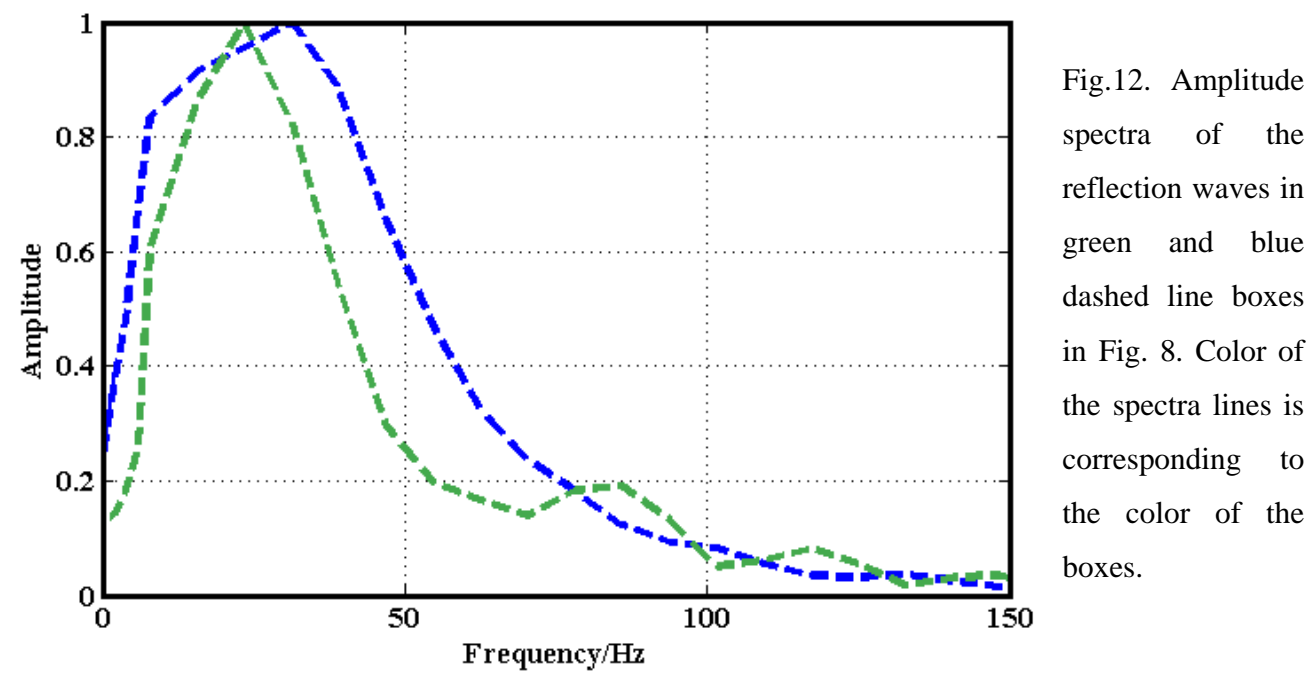

As a comparison, we illustrate another raw section (Fig. 13(a)) passing through a water well (Well-3) and its corresponding Q inversion profile in Fig. 13(b). We note that the water-bearing reservoir exhibits a relative high Q property (indicated by elliptical box in Fig. 13(b)). Fig. 13(c) shows the $\mathrm{Q}$ inversion profile obtained by traditional method. We can note that the water-bearing reservoir exhibits low Q property (indicated by elliptical box in Fig. 13(c)). We may regard this reservoir as oil-bearing reservoir. So, the traditional method gets a wrong result and we can claim that the result obtained by our method is more reasonable.

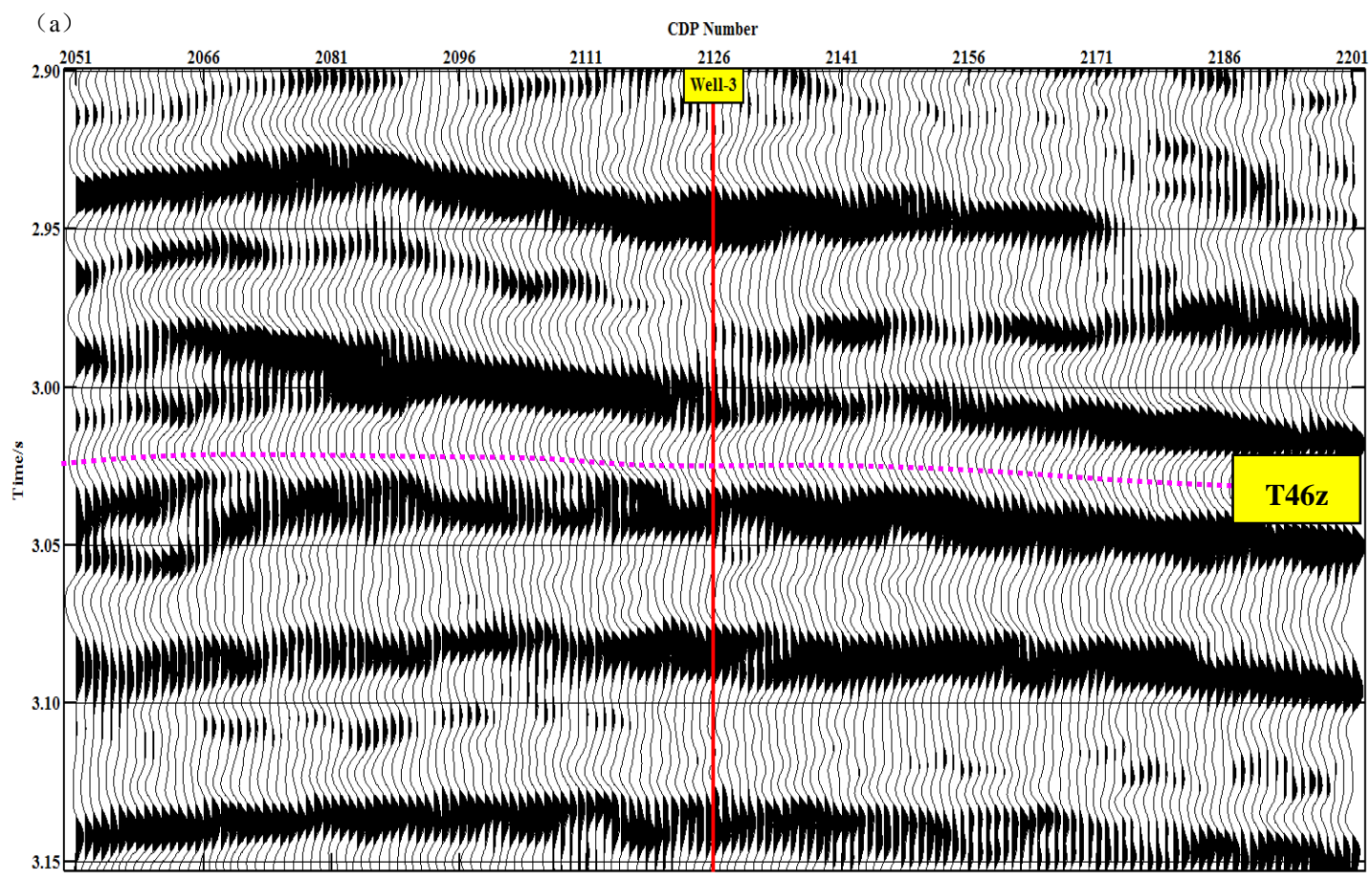




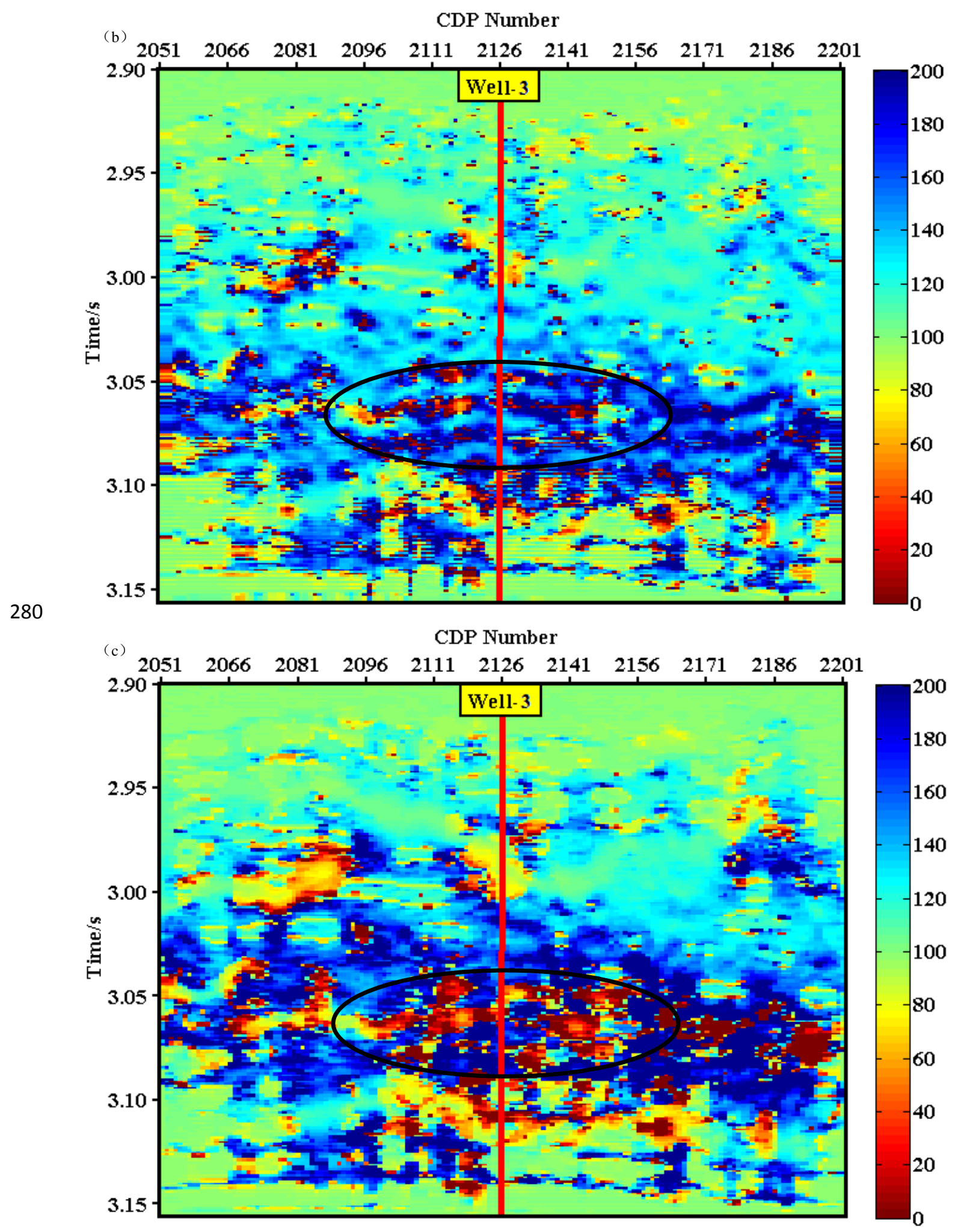

Fig. 13. (a) shows the seismic section passing through Well-3; (b) shows the inverted Q section obtained by our method; (c) shows the inverted Q section obtain by traditional method. 

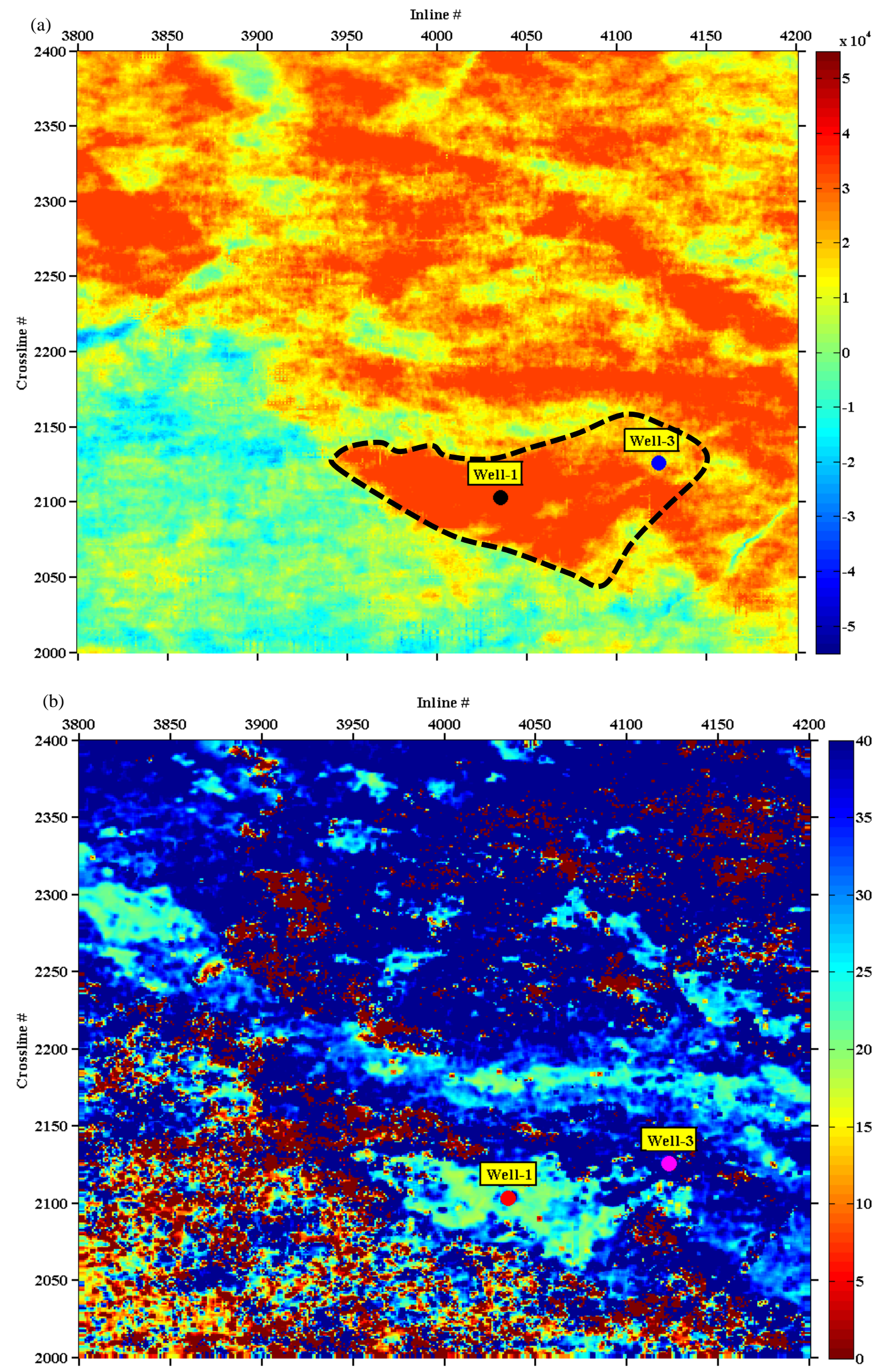

Fig. 14. Amplitude (a) and inverted Q (b) slice. The sand body is indicated by the black dashed line. 
Fig. 14(a) and (b) show the amplitude and inverted Q slice with a $16 \mathrm{~ms}$ delay from the top (T46z) reservoir, respectively. The black dashed line in Fig. 14(a) indicates the distribution of sand body around Well-1 and Well-3. Note that we have a zone of low Q value around Well-1 in Fig. 14(b), whereas Well-3 is located at a zone of high Q value. Studies by Wen et al. (2011) showed there are several faults at the southwest part of the field that cause the low energy and chaotic reflection. We suspect that the anomalously low $\mathrm{Q}$ value at the southwest part of the field is caused by the faults and fractures in this zone.

\section{Conclusions}

This paper introduces a novel algorithm for Q factor estimation. First, we introduce a recently proposed two-parameter GST to the spectral ratio method. It is more capable than the previously used time-frequency analysis technique in $\mathrm{Q}$ inversion in accommodating different non-stationary signals. Then we take the spectrum distortion caused by the window function employed during time-frequency transform into consideration and propose a new spectral-ratio $Q$ inversion formula to remove this unwanted effect. This is the major contribution of this work. In addition, a source wavelet is one of the key factors for successful Q value inversion. If we record the source signal during data acquisition, we can use the true source signal in our method directly (e.g., seismic exploration with vibrator). Otherwise, we have to preliminarily estimate the source signal. Synthetic tests demonstrate that the inverted Q results are in good agreement with the true models. Application to real data also shows that our method is capable of distinguishing between oil- and water-bearing reservoirs by using relative low $\mathrm{Q}$ attributes. And our method can get a more reasonable result than the traditional method.

\section{Acknowledgment}

We appreciate the financial support provided for the research in this paper from the National Science Foundation of China (Grant No. 41174115) and the Joint Funds of the National Natural Science Foundation of China (Grant No. U1562111).

\section{Appendix A: Equation Deduction}


$\operatorname{GST}(\tau, f)=\int_{-\infty}^{+\infty}\left[P \cdot e^{-\left[2 \pi\left(f+f_{a}\right)-2 \pi f_{d}\right]^{2} / m} e^{-i 2 \pi\left(f+f_{a}\right) t^{*}} e^{-\pi\left(f+f_{a}\right) t^{*} / Q} e^{-\frac{2 \pi^{2} f_{a}^{2}}{\lambda^{2} f^{2 p}}}\right] e^{i 2 \pi f_{a} \tau} d f_{a}$.

$P$ is independent of frequency, so it can be moved out from the integral operation. Then, we can obtain

$\operatorname{GST}(\tau, f)=P \cdot \int_{-\infty}^{+\infty} e^{-\frac{4 \pi^{2}\left(f_{a}^{2}+f^{2}+2 f a f\right)+4 \pi^{2} f_{d}^{2}-8 \pi\left(f_{a}+f\right) f_{d}}{m}-i 2 \pi\left(f_{a}+f\right) t^{*}-\frac{\pi(f a+f) t^{*}}{Q}-\frac{2 \pi^{2} f_{a}^{2}}{\lambda^{2} f^{2}+i 2 \pi f_{a}}} d f_{a}$.

315 Similarly, we move items that are independent of frequency $f_{a}$ out from the integral operation.

We can further obtain

$G S T(\tau, f)=P \cdot e^{-\left(\frac{4 \pi^{2} f^{2}+4 \pi^{2} f_{d}^{2}-8 \pi f f_{d}}{m}+i 2 \pi f t^{*}+\frac{\pi f^{*}}{Q}\right)} \int_{-\infty}^{+\infty} e^{-\left(\frac{4 \pi^{2} f_{a}^{2}+8 \pi^{2} f a f-8 \pi f_{a} f_{d}}{m}+i 2 \pi f_{a} t^{*}+\frac{\pi f a a^{*}}{Q}+\frac{2 \pi^{2} f_{a}^{2}}{\lambda^{2} f^{2}}-i 2 \pi f_{a} \tau\right)} d f_{a}$.

Eq. A. 2 can be expanded into the following type:

$\operatorname{GST}(\tau, f)=P \cdot e^{-\left(\frac{4 \pi^{2} f^{2}+4 \pi^{2} f_{d}^{2}-8 \pi f f_{d}}{m}+i 2 \pi f t^{*}+\frac{\pi f t^{*}}{Q}\right)} \cdot \int_{-\infty}^{+\infty} e^{-\left(\frac{4 \pi^{2}}{m}+\frac{2 \pi^{2}}{\left.\lambda^{2} f^{2 p}\right)}\left[f_{a}^{2}+\frac{2 f_{a}}{\frac{4 \pi^{2}}{m}+\frac{2 \pi^{2}}{\lambda^{2} f^{2 p}}}\left(\frac{4 \pi f-4 \pi f_{d}}{m}+i\left(t^{*}-\tau\right)+\frac{t^{*}}{2 Q}\right)\right]\right.} d f_{a}$

$=P \cdot e^{-\left(\frac{4 \pi^{2} f^{2}+4 \pi^{2} f_{d}^{2}-8 \pi f f_{d}}{m}+i 2 \pi f t^{*}+\frac{\pi f t^{*}}{Q}\right)} \cdot e^{\frac{\left[\left(\frac{4 \pi f-2 \sigma_{1}}{m}+i\left(t^{*}-\tau\right)+\frac{t^{*}}{2 Q}\right)\right]^{2}}{\frac{4}{m}+\frac{2}{\lambda^{2} f^{2 p}}}}$

$\cdot \int_{-\infty}^{+\infty} e^{-\left(\frac{4 \pi^{2}}{m}+\frac{2 \pi^{2}}{\lambda^{2} f^{2 p}}\right)\left[f_{a}+\frac{1}{\frac{4 \pi^{2}}{m}+\frac{2 \pi^{2}}{\lambda^{2} f^{2} p}}\left(\frac{4 \pi f-4 \pi f f_{d}}{m}+\frac{t^{*}}{2 Q}+i\left(t^{*}-\tau\right)\right)\right]^{2}} d f_{a}$.

320 Given that

$\int_{-\infty}^{+\infty} e^{-a x^{2}} d x=\sqrt{\frac{\pi}{a}}$

we can finally obtain

$\operatorname{GST}(\tau, f)=P \cdot \sqrt{\frac{1}{\frac{4 \pi}{m}+\frac{2 \pi}{\lambda^{2} f^{2 p}}}} \cdot e^{-\left[\frac{\left(2 \pi f-2 \pi f f_{d}\right)^{2}}{m}+\frac{\pi f t^{*}}{Q}\right]+\frac{\varsigma^{2}-\left(t^{*}-\tau\right)^{2}}{\frac{4}{m}+\frac{2}{\lambda^{2} f^{2 p}}}} \cdot e^{2 i\left[S\left(t^{*}-\tau\right)-\pi f t^{*}\right]}$

where $\varsigma=\frac{4 \pi f}{m}-\frac{4 \pi f_{d}}{m}+\frac{t^{*}}{2 Q}$.

\section{References}

Behura, J., Batzle, M., Hofmann R., Dorgan J., 2007. Heavy oils: Their shear story. Geophysics. 72 (5): E175-E183.

Chabyshova, E., Goloshubin, G., 2014. Seismic modeling of low-frequency "shadows" beneath gas reservoirs. Geophysics. 79 (6), D417-D423.

Cai, H.P., He, Z.H., Wang, M., Li, Y.L., He, G.M., Zou, W., 2015. A novel approach for interval inelastic absorption factor estimation. Oil Geophysical Prospecting. 50 (4), 672-677 (in Chinese). 
Chai, X., Wang, S., Yuan, S., Zhao, J., Sun, L., Wei, X., 2014. Sparse reflectivity inversion for nonstationary seismic data. Geophysics. 79 (3), V93-V105.

Chai, X., Wang, S., Wei, J., Li, J., Yin, H., 2016. Reflectivity inversion for attenuated seismic data: Physical modeling and field data experiments. Geophysics. 81 (1), T11-T24.

Chen, X.H., He, Z.H., Huang, D.J. Wen, X.T., 2009a. Low frequency shadow detection of gas reservoirs in time-frequency domain. Chinese Journal of Geophysics. 52 (1), 215-221 (in Chines).

Chen, X., 2009b. High-resolution Reservoir Architecture Analysis in Time-frequency Domain: Theory, Algorithm and Application. Ph.D. thesis, Chengdu University of Technology (in Chines).

Chen, X., He, Z., Pei X., Zhong W., Yang, W., 2013. Numerical simulation of frequency-dependent seismic response and gas reservoir delineation in turbidites: A case study from China. J. Appl. Geophys. 94 (0), 22-30.

Futterman, W.I., 1962. Dispersive Body Waves. Journal of Geophysical Research. 67 (13), $5279-5291$.

Ganley, D.C., 1981. A method for calculating synthetic seismograms which include the effects of absorption and dispersion. Geophysics. 46 (8), 1100-1107.

350 Gazdag, J., Sguazzero, P., 1984. Migration of seismic data by phase shift plus interpolation. Geophysics. 49 (2), 124-131.

Hu, W., Liu, J., Bear, L., Marcinkovich, C., 2011. A robust and accurate seismic attenuation tomography algorithm. 81th Annual International Meeting, SEG: 2727-2731.

Johnson, D., 2001. Theory of frequency dependent acoustics in patchy-saturated porous media. J.acoust. Soc. Am. 110 (2), 682-694.

Johnston, D.H., Toksöz, M.N., Timur, A., 1979. Attenuation of seismic waves in dry and saturated rocks: II. Mechanisms. Geophysics. 44 (4), 691-711.

Klimentos, T. 1995. Attenuation of P- and S-waves as a method of distinguishing gas and condensate from oil and water. Geophysics. 60, 447-458.

Li, C., Liu, X., 2015. A new method for interval Q-factor inversion from seismic reflection data. Geophysics. 80 (6), R361-R373.

Nur, A., Tosaya, C., D. Vo-Thanh., 1984, Seismic monitoring of thermal enhanced oil recovery processes. Expanded Abstracts, 54th Annual International Meeting, SEG, 337-340.

Quan, Y., Harris, J.M., 1997. Seismic attenuation tomography using the frequency shift method. Geophysics. 62 (3), 895-905.

Reine, C., M. van der Baan., Clark R., 2009. The robustness of seismic attenuation measurements using fixed- and variable-window time-frequency transforms. Geophysics. 74 (2), 
WA123-WA135.

Rickett, J.E., 2006. Method for estimation of interval seismic quality factor. U. S. Patent. $7,376,517$.

Rubino, J.G., Velis D.R., Holliger K., 2012. Permeability effects on the seismic response of gas reservoirs. Geophys. J. Int. 189 (1), 448-468.

Tonn, R., 1991. The determination of the seismic quality factor Q from VSP data: A comparison of different computational methods. Geophysical Prospecting. 39 (1), 1-27.

375 Wang, Y., 2004. Q analysis on reflection seismic data. Geophysical Research Letters 31, L17606.

Wang, Y., 2014. Stable Q analysis on vertical seismic profiling data. Geophysics. 79 (4), D217-D225.

Wang, Z.J., Hu, G.Y., Fan, T.E., Cao, S.Y., Tian, N., Lu, Q.R., 2015. Window effects for quality factor Q estimation. Oil Geophysical Prospecting. 50 (1), 118-135 (in Chinese).

Wen, X.T., He Z.H., Huang D.J. Chen, X.H., 2011. Highlight display of geological bodies based on directivity filtering. Applied Geophysics. 8 (4), 355-362.

Winkler, K.W., Nur A., 1982. Seismic attenuation: Effects of pore fluids and frictional sliding. Geophysics 47 (1), 1-15.

Zhao, W., Ge, Y., 2008. Estimation of Q from VSP data with zero offset in wavelet domain. Chinese Journal of Geophysics. 51 (4), 1202-1208 (in Chinese).

Zhou, H., Tian, Y., Ye, Y., 2014. Dynamic deconvolution of seismic data based on generalized S-transform. J. Appl. Geophys. 108 (0), 1-11. 